\title{
Laser induced damage due to scratches in the surface of nonlinear optical crystals $\mathrm{KH}_{2} \mathrm{PO}_{4}$ (KDP)
}

\author{
Dexing Zhu', Yaguo Li 1* $^{*}$, Qinghua Zhang ${ }^{1}$, Jian Wang ${ }^{1}$ and Qiao $\mathrm{Xu}^{2}$
}

\begin{abstract}
Background: Potassium dihydrogen phosphate $\left(\mathrm{KDP}, \mathrm{KH}_{2} \mathrm{PO}_{4}\right)$ crystal is an important kind of nonlinear optical crystal because of widespread use in high-intensity laser systems. A critical issue in high-intensity lasers is the laser induced damage to dielectrics since the laser fluence that optical components can stand sets an upper limit to operational fluence of laser systems.
\end{abstract}

Methods: Surface scratches on finished KDP surface were artificially generated and characterized with nano-indenter and atomic force microscope (AFM), respectively. The scratches were characterized with varied depth, directions and shapes. The morphology of scratches in different directions is quite different resulting from anisotropy of KDP crystals. The scratched surface along with surfaces free of scratches were irradiated with a $355 \mathrm{~nm}$ pulsed laser to acquire the damage threshold of KDP.

Results: The experimental results show that scratches can reduce the laser induced damage threshold (LIDT) of KDP surface by $>30 \%$. Comparing the LIDTs of scratches of different depth, directions and shapes, no significant difference is observed, but they are much lower than scratch-free surface. The morphologies vary from scratches depending on the orientation of scratches relative to crystals. Moreover, the numerical simulation of light intensity shows that cross-section shape of scratches also influences the local electrical field of incident laser light.

Conclusions: Scratches with smaller width-to-depth ratio enhance laser light intensity more significantly and more harmful to the improvement of laser damage threshold of KDP. The results suggest that the mechanical scratches must be avoided to achieve high-quality surface of strong resistance to UV laser pulses, especially those with smaller width-todepth ratios.

Keywords: Potassium dihydrogen phosphate (KDP), finite-difference time-domain (FDTD), laser induced damage threshold (LIDT)

\section{Background}

Laser-induced damage in optical materials has been a major issue for several decades since it sets the upper limit of fluence below which a laser system can be operated reliably. As frequency converters, potassium dihydrogen phosphate, $\mathrm{KDP}\left(\mathrm{KH}_{2} \mathrm{PO}_{4}\right)$, is frequently employed in large aperture laser systems because KDP is a kind of nonlinear optical material that can grow to very large dimensions [1-3]. Owing to its deliquescence, KDP is mostly finished by virtue of diamond cutting/turning under dry conditions or

\footnotetext{
* Correspondence: yargolee@163.com

${ }^{1}$ Fine Optical Engineering Research Center, Chengdu 610041, China Full list of author information is available at the end of the article
}

using cutting oil $[4,5]$. On the other hand, soft but brittle properties render KDP susceptible to scratches. Scratches generally have significant impact on damage resistance to lasers, which has been demonstrated on fused silica sample [6-9]. Thus it is of great importance to carry out investigations into the damage characteristics, in particular, the effect of scratches on laser-induced damage of KDP. Chen et al. have studied the potential effects of micro cracks on the laser induced damage of KDP [10, 11]. Using finite-difference time-domain (FDTD) algorithm, they find that light intensity of incident laser can be intensified in the vicinity of microcracks and the intensification is related to many factors, including depth, 


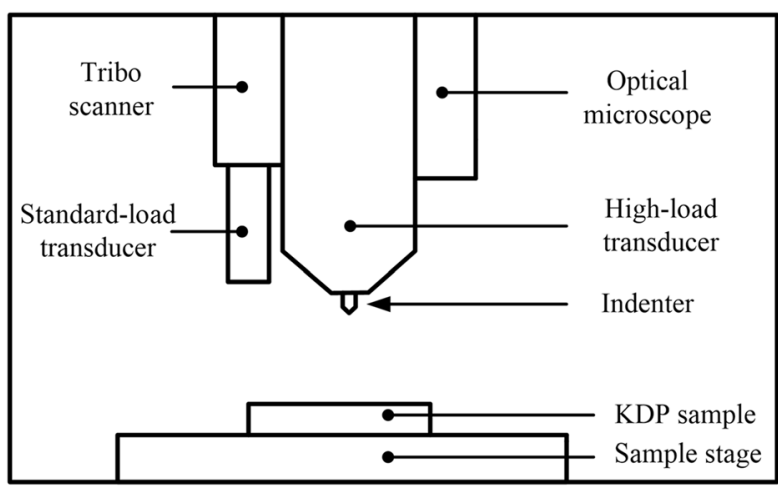

a

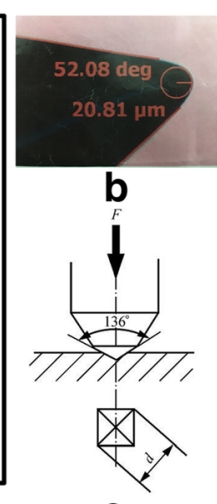

C

Fig. 1 (a) Principle of scratching experiment setup, (b) spherical indenter and (c) Vickers indenter

width and shape and the angle of microcacks relative to crystal orientation as well as the wavelength of incident laser. However, little experimental evidence accompanies their simulation and only the surfaces with brittle, ductile and free of cracks are tested for the damage performance without corroborating their simulation results about cracks of various shapes, angles, depths, etc. The influence of different scratches on laser damage other than cracks, to our knowledge, is seldom reported.

In this article, surface scratches on finished KDP surface were artificially generated and characterized with nanoindenter and atomic force microscope (AFM), respectively. The scratches were characterized with varied depth, directions and shapes. The morphology of scratches in different directions is quite different resulting from anisotropy of KDP crystals. The scratched surface along with surfaces free of scratches were irradiated with a $355 \mathrm{~nm}$ pulsed laser to acquire the damage threshold of KDP. The testing results show that scratches can reduce the LIDT of KDP surface by $>30 \%$. Comparing the LIDTs of scratches in different depth, directions and shapes, no significant difference is observed, however. In addition, the experimental phenomena are interpreted by modelling the light field around the scratches with 2-D FDTD.

\section{Methods}

\section{Scratching KDP surfaces}

A nano-indenter (TriboIndenter TI-950, Hysitron Inc., USA) is used to slide against the surface of KDP, shown in Fig. 1a. Spherical tip and Vickers tip were utilized to achieve brittle and ductile scratches of various dimensions, shown in Fig. 1b and Fig. 1c. The indenter (tip) was pressed into the surface of KDP at a preset load. After that, the crystal was translated linearly to generate a scratch. The force remained constant during the course of scratching. This way, nine scratches were produced under various conditions.
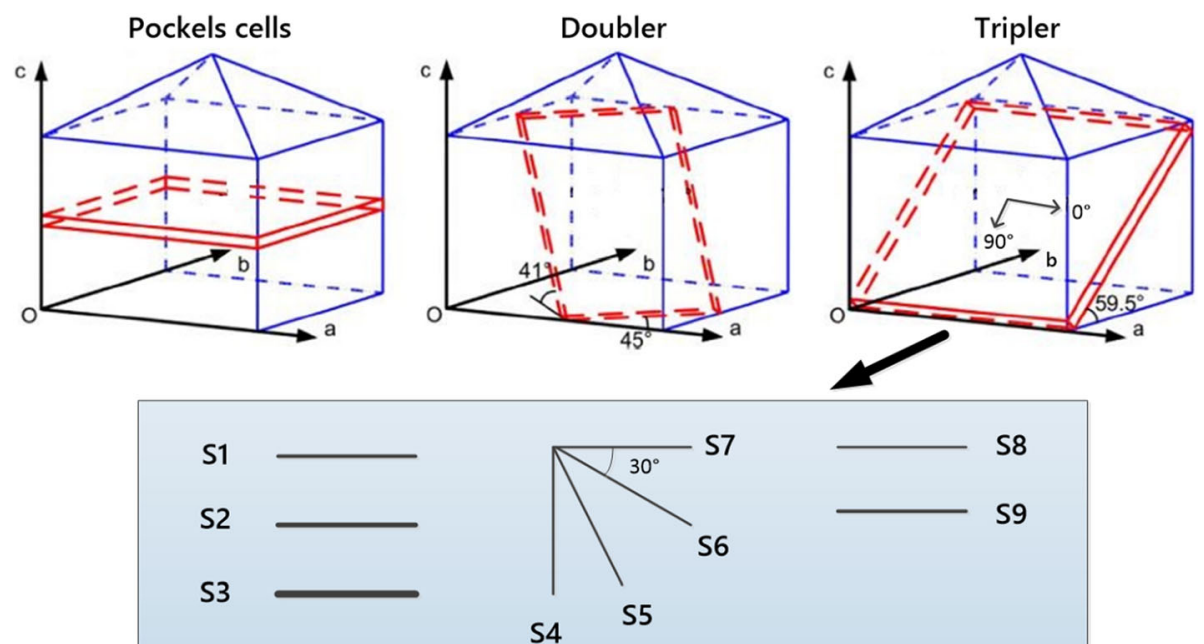

S8

S9

Fig. 2 Three kinds of KDP crystals and artificial scratches on the surface of KDP tripler 
Table 1 Parameters of the scratches

\begin{tabular}{llllllllll}
\hline & S1 & S2 & S3 & S4 & S5 & S6 & S7 & S8 & S9 \\
\hline Force $(\mathrm{mN})$ & 50 & 60 & 70 & 50 & 50 & 50 & 50 & 50 & 50 \\
Shape & ellipse & ellipse & ellipse & triangle & triangle & ellipse & ellipse & triangle & ellipse \\
Direction & $0^{\circ}$ & $0^{\circ}$ & $0^{\circ}$ & $90^{\circ}$ & $60^{\circ}$ & $30^{\circ}$ & $0^{\circ}$ & $0^{\circ}$ & $0^{\circ}$ \\
Width $(\mu \mathrm{m})$ & 10 & 11 & 12 & 8 & 9 & 10 & 10 & 8 & 10 \\
Depth $(\mathrm{nm})$ & 300 & 400 & 500 & 300 & 300 & 300 & 300 & 900 & 300 \\
Indenter & spherical & spherical & spherical & spherical & spherical & spherical & spherical & Vickers & spherical \\
W-D ratio & 33.3 & 27.5 & 24 & 26.7 & 30 & 33.3 & 33.3 & 8.9 & 33.3 \\
\hline
\end{tabular}

In high power laser systems, KDP can be used in many situations, such as Pockels cells, frequency conversion from 1 $\omega$ to $2 \omega$ (KDP doubler) and from $2 \omega$ to $3 \omega$ (KDP tripler), depending on the cutting angles of the crystal having different phase matching shown in Fig. 2 [1]. KDP tripler (uncoated) was experimented in this work, and $50 \times 50 \times 10 \mathrm{~mm}^{3}$ samples were finished by single point diamond turning. Nine artificial scratches were created on the surface of a finished KDP tripler as seen in Fig 2. S1, S2 and S3 were scratched at different loads $(50 \mathrm{mN}, 60 \mathrm{mN}, 70 \mathrm{mN})$ with a spherical indenter. S4, S5, S6 and S7 were scratched in different directions $\left(90^{\circ}, 60^{\circ}, 30^{\circ}\right.$ and $\left.0^{\circ}\right)$ at $50 \mathrm{mN}$ load. S8 and S9 were created with the Vickers and spherical indenters at the load of $50 \mathrm{mN}$. The speed of the sample stage was set to be $0.1 \mathrm{~mm} / \mathrm{s}$. The details are tabulated in Table 1 .

\section{Testing for laser damage threshold}

The setup for damage testing of the LIDT on KDP surface is sketched in Fig. 3. After the laser passes through the energy regulator and wave plate, the laser beam is converged by a focusing lens and illuminates the KDP sample residing on the sample stage. The energy detector is used to measure the pulse energy that is sampled with the splitter. The damage test of a point on the sample is completed through the computer controlled system. A laser (single longitudinal model) which runs at $355 \mathrm{~nm}$ wavelength, $\sim 400 \mu \mathrm{m}$ spot diameter $\left(1 / e^{2}\right)$ and $10 \mathrm{~ns}$ pulse duration was employed. The incident laser was normal to the surface KDP samples which were mounted at an electrically controlled stage. The smallest distance the stage can move is $0.1 \mathrm{~mm}$. The scratches were located on the front surface, i.e. entrance surface. The irradiated area was in-situ monitored with a CCD camera during the damage testing. The testing system was used for both R-on-1 and 1-on-1 testing protocols and the R:1 protocol was adopted in this article. R:1 testing determined the damage threshold by ramping the laser fluence incrementally $\left(0.25-2.5 \mathrm{~J} / \mathrm{cm}^{2}\right.$, depending on the surface to be tested) until the damage occurred and then another new site was shot again likewise. 10 sites were tested for each sample. The details can be found elsewhere $[12,13]$. The obtained 10-ns threshold was then converted into 3 ns-threshold using the empirical formula of $\tau^{0.45}$ for pulse duration of $10 \mathrm{ps} \sim 300 \mathrm{~ns}[13,14]$.

\section{Results and discussions} Morphologies of scratches

The morphologies of the nine scratches with AFM (Bruker UPTI-150, USA) are shown in Fig. 4. S1, S2 and

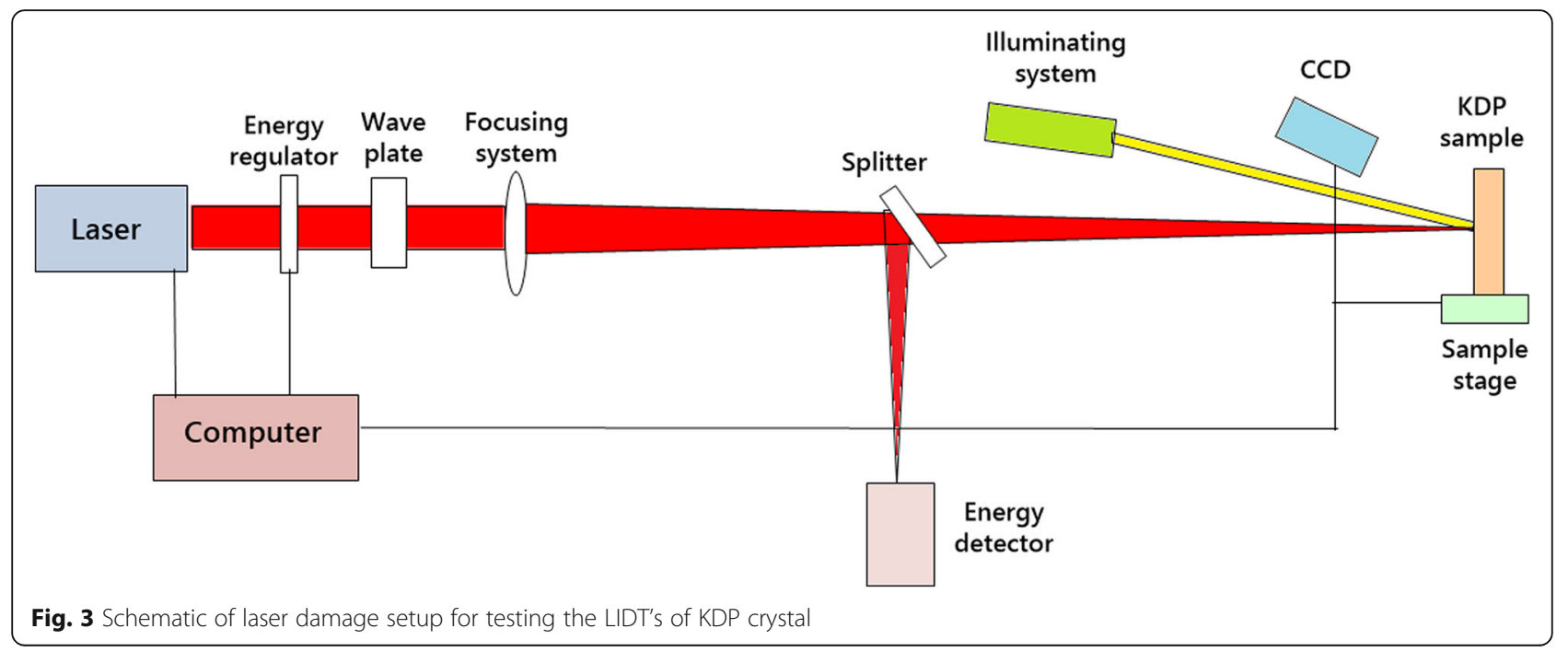



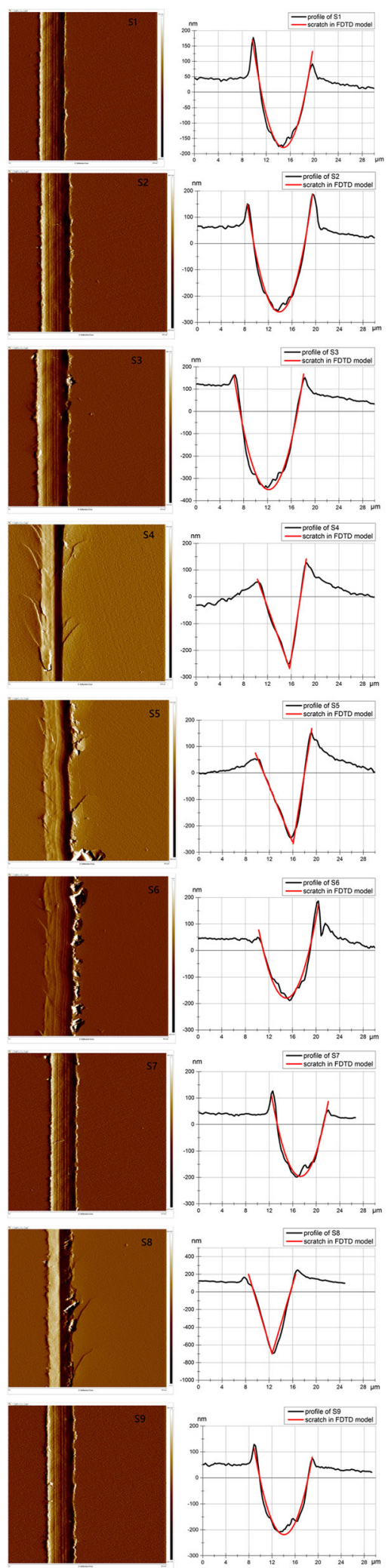

Fig. 4 Morphologies of the scratches S1 S9 characterized by AFM
S3 were cut at different loads $(50 \mathrm{mN}, 60 \mathrm{mN}, 70 \mathrm{mN})$ with the spherical indenter and the corresponding depth is about $300 \mathrm{~nm}, 400 \mathrm{~nm}, 500 \mathrm{~nm}$, respectively. Presented in Table 1 are the details of experimental conditions. It is obvious that greater cutting force results in wider and deeper scratch with spherical indenter in the same directions. As to different directions, the depth of scratches is the same but the width increases slightly from $90^{\circ}$ to $0^{\circ}$ due to anisotropy of KDP in different directions. In addition, cracks occurred at the both edges of the $90^{\circ}$ scratch while no obvious cracks can be found for the $0{ }^{\circ}$ scratch. Based on our experimental results, the fracture toughness in $90^{\circ}$ direction is less strong than in $0^{\circ}$ direction. The depth of the scratch is much deeper when the Vickers indenter was used under the same indentation load. The cross-section profile is more similar to a triangle compared to the spherical indenter and therefore the aspect ratio (depth-to-width) is far larger. We calculated the critical depth of ductile-to-brittle transition according to the formula derived by Bifano et al. $d_{c} \propto\left(\frac{E}{H}\right)$ $\left(\frac{K_{\mathrm{c}}}{\mathrm{H}}\right)^{2}$ [15]. Substituting the Young's modulus (24.0GPa), hardness $(2.0 \mathrm{GPa})$ and fracture toughness $(0.74 \mathrm{MPa} \sqrt{\mathrm{m}})$ of KDP into the formula, the estimated critical depth can be found to be $240 \sim 320 \mathrm{~nm}$ (the proportional coefficient is $0.15 \sim 0.2$ ) [15]. Because of anisotropic properties of KDP, the critical depth in various directions is accordingly different. Wang et al. reports that the critical depth ranges from $80 \mathrm{~nm}$ to $180 \mathrm{~nm}$ of critical depth [16]. In the meanwhile, the most approximate regular profiles (red lines) used in FDTD simulation in the following are also shown in Fig. 4. S4, S5, and S8 were fitted in triangular curves while the others were fitted in elliptical curves.

\section{Laser induced damage on KDP surface}

The damage threshold of scratches and scratch-free surface is presented in Fig. 5. The error bars represent the standard

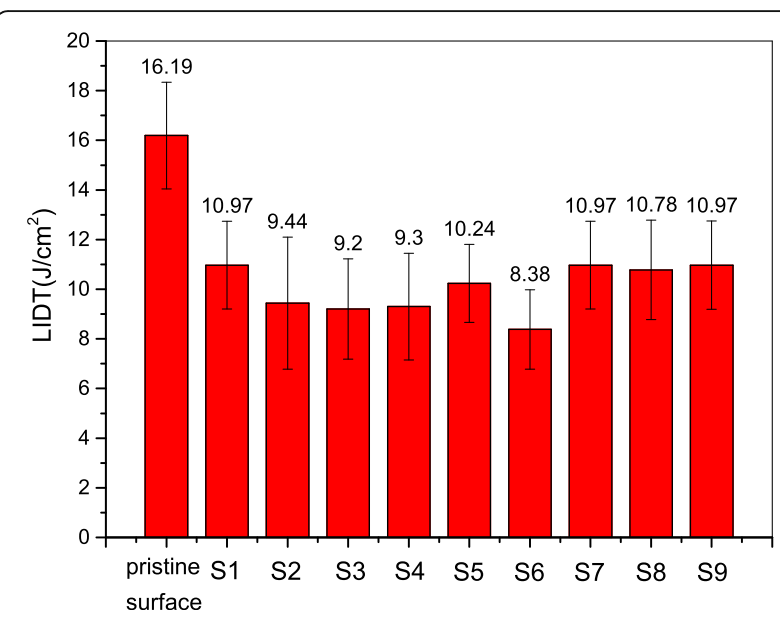

Fig. 5 The LIDTs of pristine surface and scratches on the entrance surface of KDP 

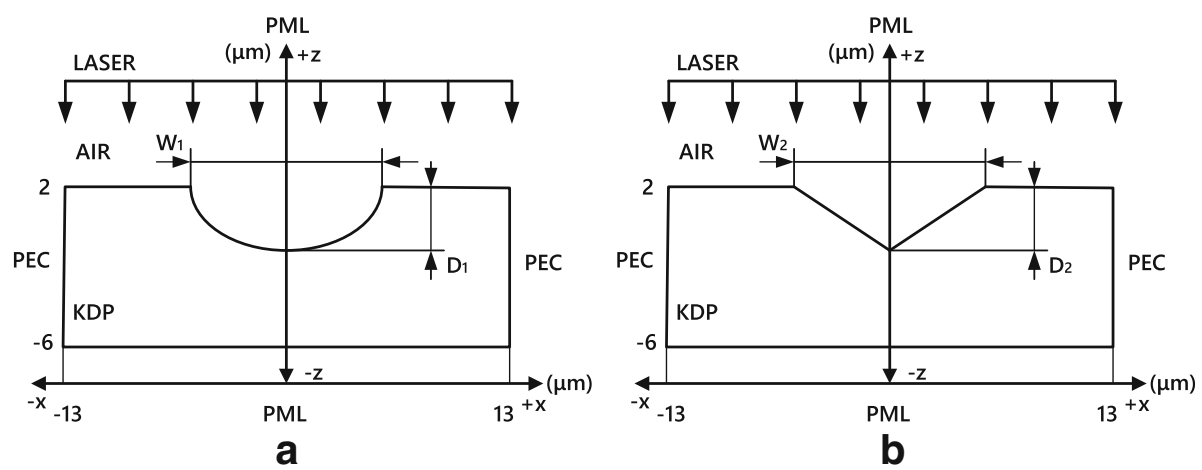

Fig. 6 2D FDTD models of surface scratches: (a) semi-oval/elliptical scratch and (b) semi-diamond/triangular scratch

deviation of testing results of 10 sites. It is apparent that the LIDT of scratch-free surface $\left(16.2 \mathrm{~J} / \mathrm{cm}^{2}\right)$ is much higher than scratches $\left(8.4 \mathrm{~J} / \mathrm{cm}^{2} \sim 11 \mathrm{~J} / \mathrm{cm}^{2}\right)$ and the threshold is lowered $>30 \%$ in the presence of scratches. The average LIDT decreases from S1 to S2 further to S3 for scratched surfaces, but there is no significant difference if the error bars are taken into account. The force to generate S3 is more than S1 and the resultant scratch is deeper and wider as well as more defects. However, these do not make a sharp difference to the LIDTs of scratches, as the experimental results show. The morphologies of scratches in different directions are quite different in Fig. 4. S4, S5 and S6 contain numerous micro-cracks relative to $\mathrm{S} 7$ but the LIDTs of these scratches are similar. The scratches created with different shaped indenters were also examined for damage threshold. No obvious different are observed in terms of LIDT of these two scratches. The triangular cross-sectioned scratch S8 is damaged at $10.78 \mathrm{~J} / \mathrm{cm}^{2}$ similar to that $\mathrm{S} 9$ slid by spherical indenter $10.97 \mathrm{~J} / \mathrm{cm}^{2}$. However, the depth of S8 $(900 \mathrm{~nm})$ is much greater than S9 $(300 \mathrm{~nm})$ and some obvious cracks are there along the scratch S8. In general, the presence of scratches would reduce the LIDT comparing to scratch-free surface by $>30 \%$, but no direct dependence of the LIDT on the scratch morphology is found from the experimental results.
Some very interesting results occurred during the laser damage test. That is, for scratch-free surface, damage sites appeared first within the bulk and then the front surface was damaged as the fluence of laser increased, which is consistent with the phenomena that $\mathrm{S}$. Reyne et al. observed [17]. By contrast, as to the scratches with apparent micro-cracks the damage mostly happened first on the scratches, e.g. scratches S4, S5, S6 \& S8. The phenomena suggest that surface damage threshold is higher than bulk for KDP and the micro-cracks on the scratches are the initiators to laser damage in our experiments. The micro-cracks must be avoided so as to achieve high damage threshold surface of KDP crystals.

\section{Damage mechanics of scratched KDP}

The influence of the shape and dimension of scratch on electric field/light intensity

2D finite-difference time-domain (FDTD) method was employed to simulate the light intensity around the surface scratches, which is helpful in understanding the possible phenomena of damage tests. Based on electromagnetic field theory, FDTD method is used to numerically solve the Maxwell's equations to obtain electric field intensity distribution. For plane wave input, the light intensity is proportional to the square of electrical field $\left(|E|^{2}\right)$, which is associated with

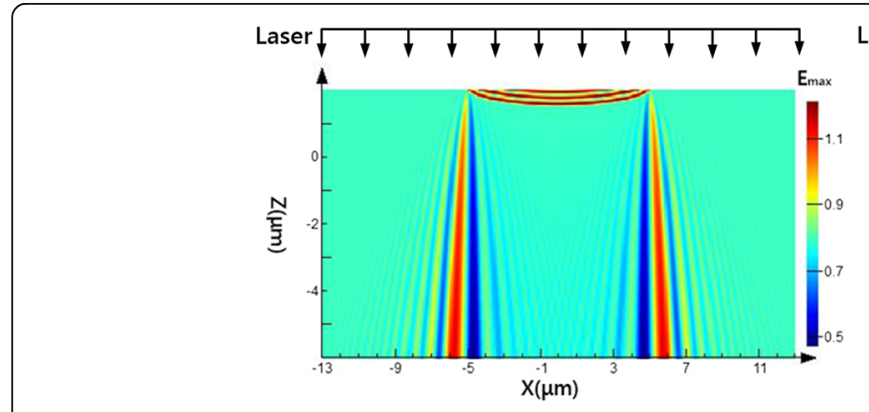

a

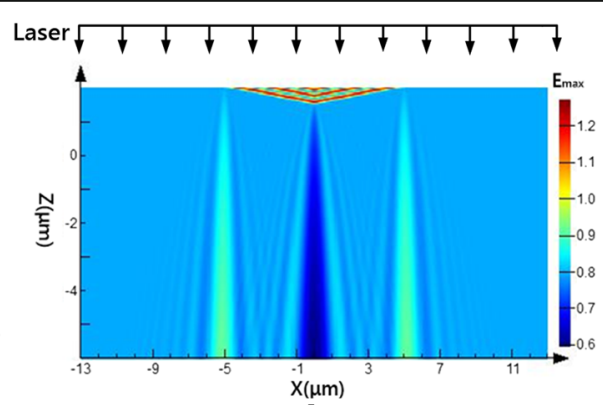

b

Fig. 7 Electric field distributions inside KDP crystal around (a) elliptical scratch and (b) triangular scratch 


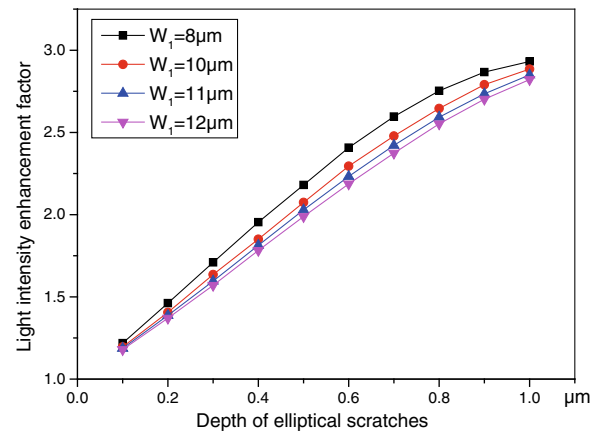

a

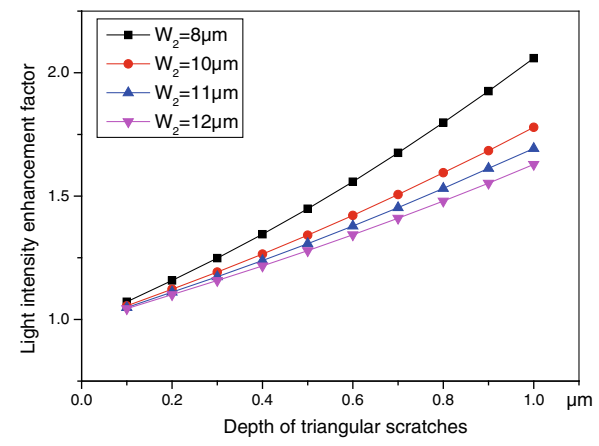

b

Fig. 8 Maximal light intensity enhancement of various dimensions. a elliptical scratches of different width and depth; (b) triangular scratches of different width and depth

the electromagnetic energy density, $1 / 2 \varepsilon_{0} \eta^{2}|E|^{2}$. Here $\varepsilon_{0}$ is the vacuum permittivity and $\eta$ is the refractive index of material. The light intensification is characterized by light intensity enhancement factor, which is uniform distribution inside a perfect KDP crystal. The amplitude of light field is responsible for non-uniform energy distribution, nonlinear increase of intense laser and consequent decline of laser damage resistance $[18,19]$. In numerical simulation, a $3 \omega$ $(\lambda=355 \mathrm{~nm})$ laser with an electric field intensity normalized to $1 \mathrm{~V} / \mathrm{m}$ was assumed to irradiate the front surface of the KDP crystal in normal direction, i.e. along the $-\mathrm{z}$ axis. A time-harmonic plane wave was adopted in this work. The simulation domain was rectangular and gridded with a uniform grid size. The grid size was $\delta=0.01 \mu \mathrm{m}$, which was less than $\lambda / 12$ to weaken the effect of numerical dispersion caused by differencing in 2D FDTD and consequently guarantee the accuracy of the simulation [11]. The perfect matched layer (PML) boundary condition was utilized along the $\mathrm{z}$ axis, and periodic boundary condition along the $\mathrm{x}$ axis [20]. KDP is a uniaxial crystal, and hence the index ellipsoid is given in the standard crystallographic coordinate system by the equation

$$
\frac{X^{2}}{n_{o}^{2}}+\frac{Y^{2}}{n_{o}^{2}}+\frac{Z^{2}}{n_{e}^{2}}=1
$$

The refractive index of KDP crystal at $\lambda=355 \mathrm{~nm}$, $T=298 \mathrm{~K}$ is $\mathrm{n}_{\mathrm{o}}=1.53, \mathrm{n}_{\mathrm{e}}=1.49$. As a result, the refractive index matrix of anisotropic KDP crystal used in this work was $\left[\begin{array}{ccc}1.53 & 0 & 0 \\ 0 & 1.53 & 0 \\ 0 & 0 & 1.49\end{array}\right]$ [21-23].

We first simulated the effects of scratch cross-section shapes on light intensity to find out whether the shape affects the electric/light field significantly. Simplified 2D models of surface scratches in our experiments (semi-oval or ellipse and semi-diamond or triangle) are illustrated in Fig. 6. The incident wave was normalized to $1 \mathrm{~V} / \mathrm{m}$ and the corresponding electric field intensity inside the crystal is $\mathrm{E}=0.7926 \mathrm{~V} / \mathrm{m}$ for a perfect surface without scratches. Figure 7 shows the electric field distributions inside the KDP crystal around two kinds of surface scratches, where width and depth of both scratches are $10 \mu \mathrm{m}$ and

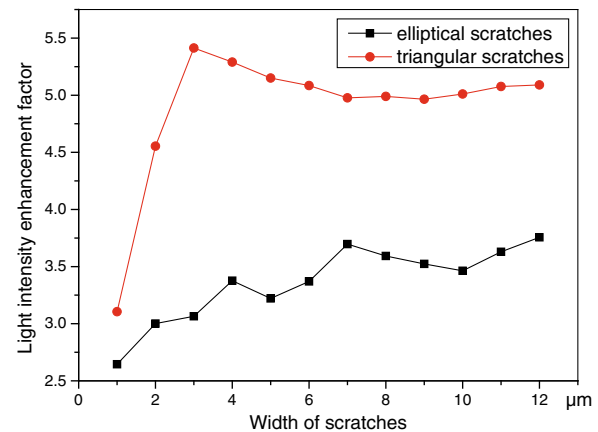

a

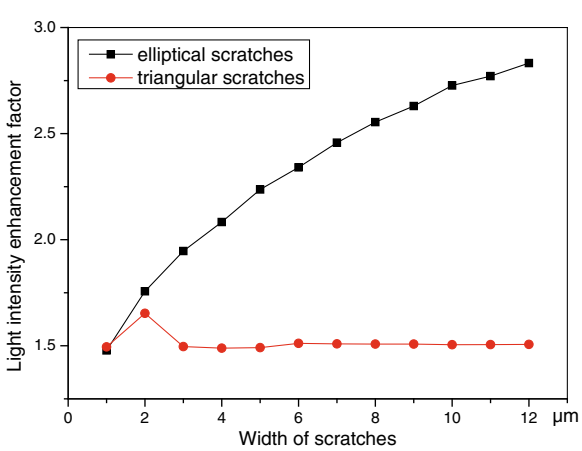

b

Fig. 9 Maximal light enhancement with respect to width of scratches for elliptical and triangular scratches with the width-to-depth ratios of (a) 2 and (b) 20 


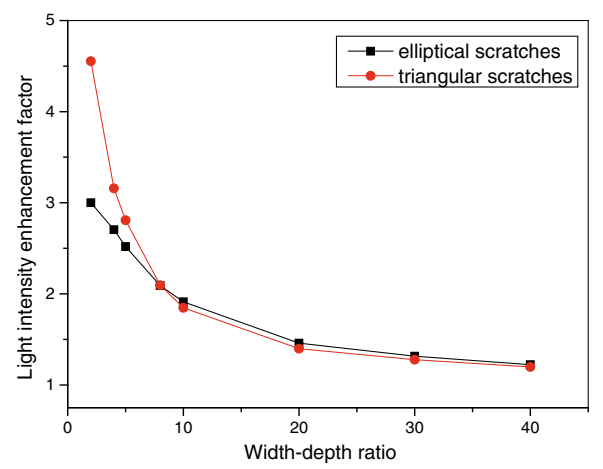

a

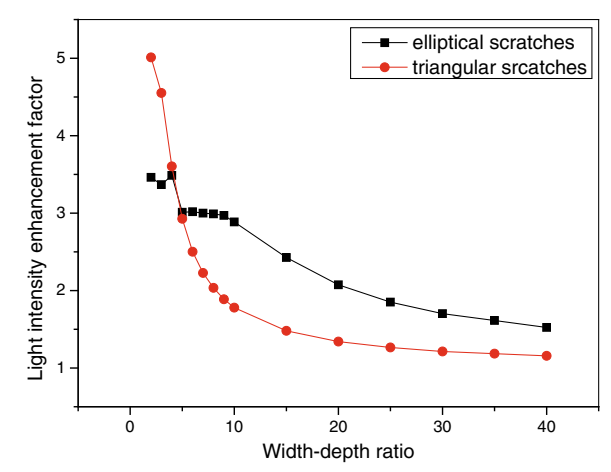

b

Fig. 10 Maximal light enhancement with respect to width-to-depth ratio for elliptical and triangular scratches of (a) $2 \mu \mathrm{m}$ and (b) $10 \mu \mathrm{m}$ wide
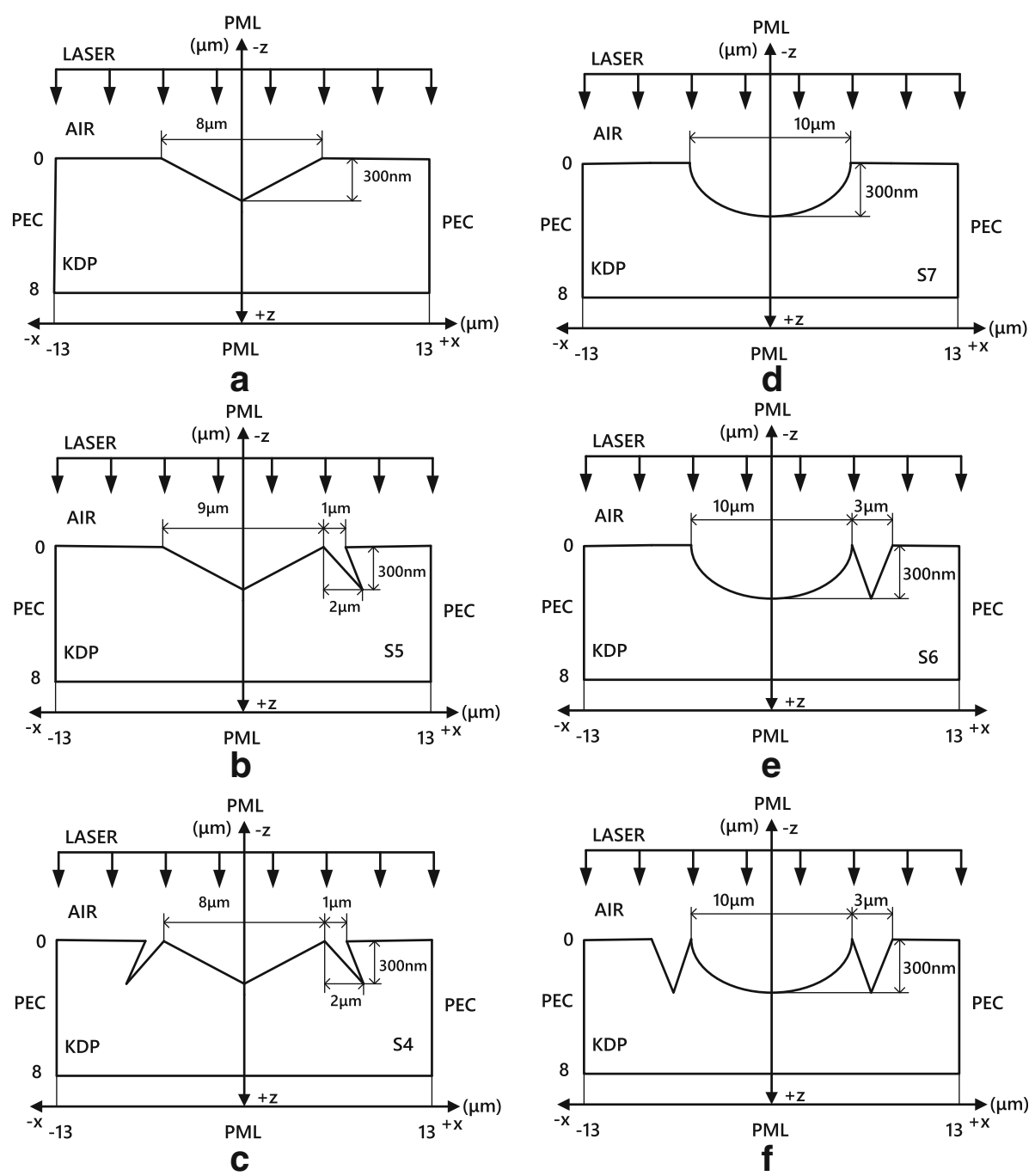

Fig. 11 FDTD models of scratches created in different crystal orientations 
$500 \mathrm{~nm}$. One can see that electric field distributions are similar for two surface scratches and the electric field intensity of the region directly under the scratch for both scratches is relatively low $(0.6 \mathrm{~V} / \mathrm{m}$ vs $0.8 \mathrm{~V} / \mathrm{m}$ ) and electric field is enhanced at two flanks of the scratches $(1.1 \mathrm{~V} / \mathrm{m}$ vs $0.8 \mathrm{~V} / \mathrm{m})$ and symmetrically distributed. The causes are that the transmitting wave through the surface scratch overlaps with the incident wave inside the crystal and they constructively interfere with each other. The light intensity enhancement factor inside KDP crystal for two scratches in simulated region was extracted to be 2.07 and 1.34, respectively, the maximal electric field intensity around elliptical scratch 1.24 times that of triangular one.

The maximal light intensity in investigated region for various dimensions (width, depth) was modelled in Fig. 8. For both elliptical and triangular scratches, the maximal light intensity due to scratches increases with the depth of the scratches and decreasing the width will also result in the enhancement of light intensity. The enhancement of light intensity by elliptical scratches seems more remarkable when the depth and width of elliptical and triangular scratches are similar. For instance, for $1.0 \mu \mathrm{m}$ deep, $8 \mu \mathrm{m}$ wide scratches, the light enhancement can be 2.93 times incident light for elliptical scratches and 2.05 times for triangular scratches. According to the simulation results, an elliptical scratch with $10 \mu \mathrm{m}$ width and $300 \mathrm{~nm}$ depth (like S1) would cause 1.6 times light intensity enhancement, while 1.8 times for S2 and 2.0 times for S3. S8 would cause 1.9 times light enhancement, and S9 would cause 1.6 times light enhancement. As a result, scratches can reduce the LIDT of KDP surface by $>30 \%$. But the difference of light enhancement among scratches is not large enough to make a significant difference to the LIDTs of scratches, as observed in the experiment.

Figure 9 shows the maximal light intensity enhancement for two kinds of scratches with respect to the width of scratches when width-to-depth ratio keeps constant. The width-to-depth ratios are 2 and 20, respectively. It can be seen from Fig. 9b that the maximal light enhancement is more significant for elliptical scratches than triangular ones for the ratio of 20. This discrepancy increases with the width of scratches. The light enhancement is nearly linearly proportional to the width of scratches while it is almost unchanged for triangular scratches when the width of scratches exceeds $3 \mu \mathrm{m}$ in the modeled situations. By contrast, the triangular ones affect the light intensity more than elliptical ones in the case of width-to-depth ratio 2 . The critical width-to-depth ratio is dependent on the width of scratches, see below. For micro-cracks usually with width-to-depth far much less than 1, the light field intensification is more severe than those with rounded tips.
The light enhancement of various width-to-depth ratios was also simulated in Fig. 10. The scratches with two sorts of width $2 \mu \mathrm{m}$ and $10 \mu \mathrm{m}$ were numerically modelled. The maximal light enhancement decreases with the width-to-depth ratio for both kinds of scratches. When width-depth ratio is less than 5 for $10 \mu \mathrm{m}$ wide scratch, maximal light enhancement caused by triangular scratches is more pronounced than elliptical ones. However, the enhancement is relative less strong for triangular than elliptical scratches as the ratio is in excess of 5 . On the other hand, $2 \mu \mathrm{m}$ scratch with triangular shape plays a more important role than elliptical scratch as width-to-depth ratio is $<8$.

\section{Effect of cracks on scratch on light enhancement}

The scratches in different crystal orientations under the same conditions feature different morphology. Some are full of micro-cracks but some contain few cracks. Thus we imposed microcracks onto scratches to simulate the light enhancement as shown in Fig. 11. The maximum of light intensity inside KDP crystal modulated by the scratches was extracted in Fig. 12. From the results, the cracks at the edges of scratches would cause higher light intensity. The light enhancement is increased from 1.24 to 1.98 and 1.99 for triangular scratches with cracks and from 1.6 to 2.55 and 2.56 for elliptical scratches. Mirco-cracks make a big difference to light enhancement, but the number microcracks seem to have little influence on light enhancement. According to the simulation results, the light intensity enhancement factors caused by S4, S5, S6, and S7 would be $2.56,2.55,2.55$, and 1.6, respectively. However, no significant difference among the LIDTs of scratches was observed in the experiment. There may be two reasons: the differences on light enhancement are of small difference to make a significant influence on the LIDTs of different

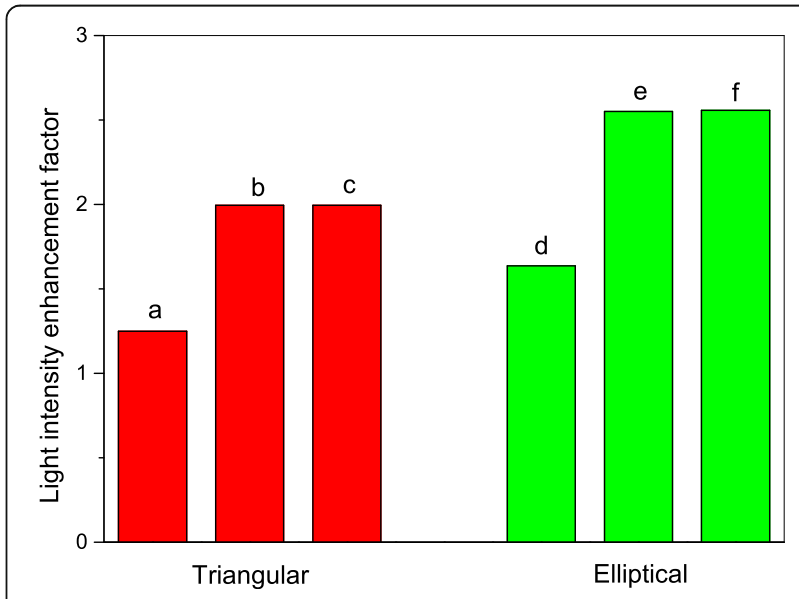

Fig. 12 The maximum of light intensity modulated by the scratches along with microcracks 
scratches in our experiments and other factors may be dominant.

\section{Conclusions}

Surface scratches of various features were artificially generated and damaged with UV laser. The effect of scratches on laser damage was investigated there. It is found that the morphologies vary from scratches depending on the orientation of scratches relative to crystals. That in $90^{\circ}$ direction is full of micro-cracks while the scratch in 0 direction contains few micro-cracks. The causes are the anisotropy of KDP. All the laser damage thresholds of scratches are much lower than scratch free surface, but no obvious dependence of the LIDT on the scratch morphology is found. Moreover, the numerical simulation of light intensity shows that cross-section shape of scratches also influences the local electrical field of incident laser light. Scratches with smaller width-todepth ratio enhance laser light intensity more significantly and more harmful to the improvement of laser damage threshold of KDP. The results suggest that the mechanical scratches must be avoided to achieve high-quality surface of strong resistance to UV laser pulses, especially those with smaller width-to-depth ratios.

\section{Abbreviations}

AFM: Atomic force microscope; FDTD: Finite-difference time-domain; KDP: Potassium dihydrogen phosphate; LIDT: Laser induced damage threshold; PML: Perfect matched layer

\section{Acknowledgements}

The damage testing by Dr. Yi Zheng, Mr. Zhichao Liu, and Mr. Qi Lin of Fine Optical Engineering Research Center is thankfully appreciated.

\section{Funding}

$Y L$ gratefully acknowledges the funding of the he Foundation for the Development of Science \& Technology of China Academy of Engineering Physics (2015B0203032), the National Natural Science Foundation of China (No.51505444), the Foundation of the Laboratory of Precision Manufacturing Technology of China Academy of Engineering Physics (ZZ15004); DZ acknowledges the funding of Science Challenge Project (No. JCKY2016212A506-0503).

\section{Availability of data and materials}

All datasets on which the conclusions of the manuscript rely are included in the manuscript.

\section{Authors' contributions}

DZ conducted the experiments on LIDT experiments of KDP. YL proposed the idea of the LIDT at the scratches on KDP. QZ proposed to study the LIDT of KDP. JW was responsible for providing KDP samples used in experiments. QX is in charge of the whole research project. All the authors have approved the final manuscript for publication.

\section{Ethics approval and consent to participate}

Not applicable.

\section{Consent for publication}

Not applicable.

\section{Competing interests}

The authors declare that they have no competing interests.

\section{Publisher's Note}

Springer Nature remains neutral with regard to jurisdictional claims in published maps and institutional affiliations.

\section{Author details}

${ }^{1}$ Fine Optical Engineering Research Center, Chengdu 610041, China.

${ }^{2}$ Research Center of Laser Fusion, China Academy of Engineering Physics, Mianyang 621900, China.

Received: 12 August 2017 Accepted: 27 October 2017

Published online: 06 November 2017

\section{References}

1. Baisden, P.A., Atherton, L.J., Hawley, R.A., Land, T.A., Menapace, J.A., Miller, P. E., et al.: Large optics for the National Ignition Facility. Fusion Sci. Technol. 69, 295-351 (2016)

2. Campbell, J.H., Hawley-Fedder, R.A., Stolz, C.J., Menapace, J.A., Hackel, M.R.B., Whitman, P.K., et al.: NIF optical materials and fabrication Technologies : an overview. Proc. SPIE. 5341, 84-101 (2004)

3. Demos, S.G., DeMange, P., Negres, R.A., et al.: Investigation of the electronic and physical properties of defect structures responsible for laser-induced damage in DKDP crystals. Opt. Express. 18, 13788-13804 (2010)

4. Namba, Y., Katagiri, M., Nakatsuka, M.: Single point diamond turning of KDP inorganic nonlinear crystals for laser fusion. J. Japan Soc. Precis. Eng. 64, 1487-1491 (1998) (in Japanese)

5. Fuchs, B.A., Hed, P.P., Baker, P.C.: Fine diamond turning of KDP crystals. Appl. Opt. 25, 1733-1735 (1986)

6. Li, Y., Yuan, Z., Wang, J., Xu, Q.: Laser-induced damage characteristics in fused silica surface due to mechanical and chemical defects during manufacturing processes. Opt. Laser Technol. 91, 149-158 (2017)

7. Li, Y., Ye, H., Yuan, Z., Liu, Z., Zheng, Y., Zhang, Z., et al.: Generation of scratches and their effects on laser damage performance of silica glass. Sci Rep. 6, 34818 (2016)

8. Wang, J., Li, Y., Yuan, Z., Ye, H., Xie, R., Chen, X., et al.: Producing fused silica optics with high UV-damage resistance to nanosecond pulsed lasers. Proc. SPIE. 9532, 95320H (2015)

9. Genin, F.Y., Salleo, A., Pistor, T.V., et al.: Role of light intensification by cracks in optical breakdown on surfaces. J. Opt. Soc. Amer. A. 18, 2607-2616 (2001)

10. Cheng, J., Chen, M., Liao, W., Wang, H., Wang, J., Xiao, Y., et al.: Influence of surface cracks on laser-induced damage resistance of brittle $\mathrm{KH}_{2} \mathrm{PO}_{4}$ crystal. Opt. Express. 22, 28740-28755 (2014)

11. Chen, M., Cheng, J., Li, M., Xiao, Y.: Study of modulation property to incident laser by surface micro-defects on $\mathrm{KH}_{2} \mathrm{PO}_{4}$ crystal. Chinese Phys. B. 21, 064212 (2012)

12. Ye, H., Li, Y., Zhang, Q., Wang, W., Yuan, Z., Wang, J., et al.: Post-processing of fused silica and its effects on damage resistance to nanosecond pulsed UV lasers. Appl. Opt. 55, 3017-3025 (2016)

13. Ye, H., Li, Y., Yuan, Z., Wang, J., Yang, W., Xu, Q.: Laser induced damage characteristics of fused silica optics treated by wet chemical processes. Appl. Surf. Sci. 357, 498-505 (2015)

14. Bude, J., Miller, P., Baxamusa, S., Shen, N., Laurence, T., Steele, W., et al.: High fluence laser damage precursors and their mitigation in fused silica. Opt. Express. 22, 5839-5851 (2014)

15. Bifano, T.G., Dow, T.A., Scattergood, R.O.: Ductile-regime grinding: a new Technology for Machining Brittle Materials. Trans. ASME J. Eng. Ind. 113, 184-189 (1991)

16. Wang, S., An, C., Zhang, F., Wang, J., Lei, X., Zhang, J.: An experimental and theoretical investigation on the brittle ductile transition and cutting force anisotropy in cutting KDP crystal. Int J Mach Tool Manu. 106, 98-108 (2016)

17. Reyné, S., Duchateau, G., Hallo, L., et al.: Multi-wavelength study of nanosecond laser-induced bulk damage morphology in KDP crystals. Appl. Phys. A Mater. Sci. Process. 119 1317-1326 (2015)

18. Cheng, J., Chen, M., Liao, W., Wang, H., Xiao, Y., Li, M.: Fabrication of spherical mitigation pit on $\mathrm{KH}_{2} \mathrm{PO}_{4}$ crystal by micro-milling and modeling of its induced light intensification. Opt.Express. 21, 16799-16813 (2013)

19. Stolz, C.J., Feit, M.D., Pistor, T.V.: Laser intensification by spherical inclusions embedded within multilayer coatings. Appl. Opt. 45, 1594-1601 (2006)

20. Schneider, J.B. Understanding the Finite-Difference Time-Domain Method, (2014)

21. Boyd, R.W: Nonlinear Optics $3^{\text {rd }}$ Ed., Academic Press. http://www. sciencedirect.com/science/book/9780123694706 (2008) 
22. Dmitriev, VG, Gurzadyan, GG, Nikogosyan, DN: Handbook of Nonlinear Optical Crystals, Springer. http://www.springer.com/us/book/9783540653943 (1999)

23. Zhu, L., Zhang, X., Xu, M., Liu, B., Ji, S., Zhang, L., Zhou, H., Liu, F., Wang, Z., Sun, $X$ : Refractive indices in the whole transmission range of partially deuterated KDP crystals. AIP Adv. 3, 112114 (2013)

\section{Submit your manuscript to a SpringerOpen ${ }^{\bullet}$} journal and benefit from:

- Convenient online submission

- Rigorous peer review

- Open access: articles freely available online

- High visibility within the field

- Retaining the copyright to your article

Submit your next manuscript at $\gg$ springeropen.com 